\title{
Comparison of Needle Aspiration with Incision and Drainage for the Treatment of Peritonsillar Abscess
}

\author{
T-H Chi ${ }^{1,2}$, C-H Yuan ${ }^{2}$, Y-H Tsao ${ }^{1}$
}

\begin{abstract}
Background: Peritonsillar abscess is a common clinical condition encountered in the practice of otolaryngology. However, there is no consensus yet in terms of best treatment of peritonsillar abscess. The current prospective clinical study was performed to compare two common treatments of peritonsillar abscess, namely, needle aspiration versus incision and drainage for peritonsillar abscess Methods: A prospective study was performed on patients with diagnosis of peritonsillar abscess from January 2008 to December 2012. A total of 110 patients were enrolled in this study and were randomly allocated to two groups who were treated with needle aspiration and incision and drainage, respectively. Data collected included age, gender, clinical symptoms and treatment outcome.

Results: There were 98 males and 12 females ranging from 12 to 79 years of age, with mean age of 31.0 \pm 15.0 years. Complete remission of symptoms was achieved for all patients during the follow-up. No significant difference was observed regarding baseline characteristics including age, gender, location of the abscess, and duration of symptoms between the two groups. Pain scores taken one and 24 hours after either procedure suggested that patients treated with needle aspiration felt less pain compared with those treated with incision and drainage. Additionally, no significant difference in the median hospital stay was found between the two groups. There were two (3.6\%) patients in the needle aspiration group who required incision and drainage after two needle aspirations. No significant side effects were observed in either group.

Conclusions: The complication rate and length of hospital stay are similar between the needle aspiration and the incision and drainage groups. Needle aspiration is an efficient and safe procedure to treat peritonsillar abscess. Furthermore, needle aspiration was found to be superior to incision and drainage in terms of the post-procedure pain score for the patients. It is suggested that needle aspiration can be performed as the first-line treatment of peritonsillar abscess.
\end{abstract}

Keywords: Incision and drainage, needle aspiration, peritonsillar abscess

WIMJ Open 2014; 1 (1): 11

\section{INTRODUCTION}

Peritonsillar abscess, namely quinsy, is defined as a collection of pus in the space between the capsule of the tonsil and the superior pharyngeal constrictor muscle. It is most commonly found in young adults and adolescents, and less seen in children. Although peritonsillar abscess was regarded as the complication of acute tonsillitis in the past, it is now considered to be secondary to infection of Weber's gland located in the upper tonsillar pole (1). The pathogens associated with peritonsillar abscess are often polymicrobial. The most pre-

From: ${ }^{1}$ Department of Otolaryngology, Taoyuan Armed Forces General Hospital, Taoyuan, Taiwan and ${ }^{2}$ Department of Otolaryngology, Kaohsiung Armed Forces General Hospital, Kaohsiung, Taiwan.

Correspondence: Dr C-H Yuan, Department of Otolaryngology, Kaohsiung Armed Forces General Hospital, 2 Chung Cheng 1st Road, Kaohsiung, 802, Taiwan, Republic of China. E-mail: cooljackychi@yam.com dominant organisms are aerobic Streptococci, as well as anaerobic Prevotella and Peptostreptococci (2). Peritonsillar abscess is the most common type of deep neck infection. However, the management is still in debate. In the present study, a series of 110 patients with peritonsillar abscesses treated at a regional hospital in Taiwan was prospectively studied. The aim of this study was to compare the outcomes of two common approaches, namely needle aspiration and incision followed by drainage, and propose a treatment protocol.

\section{SUBJECTS AND METHODS}

Patients with peritonsillar abscess were enrolled in this study from January 2008 to December 2012. All patients received a comprehensive diagnostic procedure including medical history taking, physical and laboratory examinations. Fibre- 
optic endoscopy was performed to exclude other lesions. Patients were randomly divided into two groups with group A $(n=55)$ treated with needle aspiration, whereas group B (n $=55)$ was treated with incision and drainage.

In group $\mathrm{A}$, needle aspiration was performed using one $10 \mathrm{ml}$ syringe with a 22-gauge needle at the site of maximum bulge above the upper pole of the tonsil under local anaesthesia with 10\% lidocaine (Xylocaine) spray. For group $\mathrm{B}$, one incision was made about 1 to $2 \mathrm{~cm}$ at the site between the base of the uvula and upper third molar tooth under local anaesthesia with submucous infiltration of $2 \%$ lidocaine (Xylocaine). Following either procedure, all patients were prescribed intravenous fluid hydration and empiric parenteral antibiotics of amoxicillin/clavulanate. The pus samples from all patients were sent for culture.

Intensity of pain following either procedure was assessed using a visual analogue scale (VAS) with a $100-\mathrm{mm}$ line from zero, as no pain, to 10, as the worst pain. The score of pain intensity was acquired at one, 24 and 48 hours after the procedure.

The factors included for analysis were age, gender, clinical symptoms and treatment outcome. Continuous variables were summarized as means \pm standard deviation and were analysed with the Student's $t$-test. Categorical variables were summarized as percentages and were analysed using the Chi-square test. A $p$ value $<0.05$ was considered to be statistically significant. All statistical analyses were per- formed using SPSS version 17.0 for Windows (SPSS, Inc., Chicago, Illinois).

\section{RESULTS}

There were 110 patients with peritonsillar abscess enrolled in this study, comprising 98 males $(89.1 \%)$ and 12 females $(10.9 \%)$ with a male:female ratio of $8.2: 1$. The mean age was $31.0 \pm 15.0$ years, with a range of 12 to 79 years. About $80 \%$ $(88 / 110)$ of the patients were younger than 40 years old. All patients complained of sore throat and had unilateral peritonsillar abscesses, including $52.7 \%$ (58/110) with the lesions on the right side and $47.3 \%$ (52/110) on the left. Other symptoms included odynophagia ( $\mathrm{n}=101 ; 91.8 \%$ ), dysphagia $(n=60 ; 54.5 \%)$, uvula deviation $(n=45 ; 40.9 \%)$, trismus $(\mathrm{n}=37 ; 33.6 \%)$ and fever $(\mathrm{n}=36 ; 32.7 \%)$. The mean duration of symptoms prior to admission was $4.7 \pm 2.8$ days (range 1-14 days) for the needle aspiration group and $4.3 \pm$ 2.5 days (range 1-14 days) for the incision and drainage group. No statistical difference was found regarding baseline characteristics including age, gender, affected side and duration of symptoms between groups A and B (Table 1).

The post-procedure pain intensity scores taken at one and 24 hours were significantly lower in group A compared to group $\mathrm{B}(p<0.05)$. However, no significant differences were found with the scores taken at 48 hours after the procedures (Table 2). In group A, 49 patients (89.1\%) were cured with a single needle aspiration, and four patients $(7.3 \%)$ needed an additional needle aspiration. Two patients

Table 1: Baseline characteristics of the patients with peritonsillar abscess

\begin{tabular}{|c|c|c|c|}
\hline Characteristics & $\begin{array}{l}\text { Group A (needle aspiration) } \\
\qquad n=55\end{array}$ & $\begin{array}{l}\text { Group B (incision and drainage) } \\
\qquad n=55\end{array}$ & $p$-value \\
\hline Gender & & & $0.541^{\mathrm{a}}$ \\
\hline Male & $48(87.3 \%)$ & $50(90.9 \%)$ & \\
\hline Female & $7(12.7 \%)$ & $5(9.1 \%)$ & \\
\hline Age in years & $30.0 \pm 14.5$ & $32.1 \pm 15.5$ & $0.470^{\mathrm{b}}$ \\
\hline Affected side & & & $0.251^{\mathrm{a}}$ \\
\hline Right & 32 & 26 & \\
\hline Left & 23 & 29 & \\
\hline $\begin{array}{l}\text { Duration of symptoms prior } \\
\text { to admission in days }\end{array}$ & $4.7 \pm 2.8$ & $4.3 \pm 2.5$ & $0.479^{b}$ \\
\hline
\end{tabular}

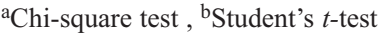

Table 2 Treatment outcome of the patients with peritonsillar abscess

\begin{tabular}{lccc}
\hline Parameters & $\begin{array}{c}\text { Group A (needle aspiration) } \\
\mathbf{n}=\mathbf{5 5}\end{array}$ & $\begin{array}{c}\text { Group B (incision } \\
\text { and drainage) } \mathbf{n}=\mathbf{5 5}\end{array}$ & p-value $^{\mathbf{a}}$ \\
\hline $\begin{array}{l}\text { Pain intensity, hours after } \\
\text { procedure }\end{array}$ & & & \\
1 & $4.5 \pm 0.8$ & $5.3 \pm 1.1$ & $0.000^{*}$ \\
24 & $2.9 \pm 0.8$ & $3.9 \pm 0.9$ & $0.000^{*}$ \\
48 & $1.9 \pm 0.6$ & $2.1 \pm 0.7$ & 0.322 \\
Hospital stay in days & $4.4 \pm 1.7$ & $4.6 \pm 1.6$ & 0.488 \\
\hline
\end{tabular}

aStudent's $t$-test, ${ }^{*} p<0.05$ 
(3.6\%) were not cured with two needle aspirations and then received incision and drainage. In group B, all patients were cured with a single incision and drainage $(100.0 \%)$. There was no statistical difference found in either mean duration of hospital stay: $4.4 \pm 1.7$ days in group A versus $4.6 \pm 1.6$ days in group B (Table 2). No complications were seen in either group.

\section{DISCUSSION}

Peritonsillar abscess is frequently seen in otolaryngologic emergency. If not treated promptly and adequately, it may give rise to severe complication such as airway compromise. Despite frequent encountering of peritonsillar abscess in clinical practice, there is a lack of consensus on its definite treatment.

According to the report by Khan et al (3), most of the patients with peritonsillar abscess in Pakistan were aged between 20 and 40 years $(76.7 \%)$ and predominantly male (71.4\%). Most of the patients had unilateral peritonsillar abscess, and the reported occurrence rate for the bilateral peritonsillar abscess was about $4.9 \%$ (4). Sore throat and odynophagia were the main presenting symptoms. In the current study, we found that patients with peritonsillar abscess were more likely to be younger than 40 years of age and the majority of the patients were male as well. All patients in the current study had unilateral peritonsillar abscess. However, there was no obvious predominant side for the abscess.

The principal treatment of peritonsillar abscess includes empiric broad-spectrum antibiotics and drainage of the pus (5). As far as the drainage of peritonsillar abscess was concerned, quinsy tonsillectomy, needle aspiration, incision and drainage were the treatment strategies. Through the years, needle aspiration as well as incision and drainage have become the mainstream treatment strategies.

In the present study, needle aspiration caused significantly less pain for the patients at one and 24 hours after the procedure. This may be due to the simplicity and less invasiveness of the needle aspiration procedure. However, as patients treated with either procedure gradually recovered, the pain scores taken at 48 hours after either procedure did not show statistically significant differences.

All patients in the incision and drainage group were cured without failure in our study. In the needle aspiration group, nearly $90 \%$ of patients were cured with only one procedure, and $3.6 \%$ of the patients needed one more needle aspiration and subsequent incision and drainage. An earlier study from India by Tyagi et al reported that $86.1 \%$ of the patients $(31 / 36)$ were healed with one needle aspiration and $2.8 \%(1 / 36)$ of the patients required further incision and drainage (6). The current study showed similar results with a larger sample size. In contrast, Khan et al (7) demonstrated that the patients who received incision and drainage had shorter hospital stay when compared with those who received needle aspiration. However, such difference was not observed in the current study.

Although needle aspiration has a slightly higher failure rate, it provided advantages such as less pain to the patients $(6,8)$. Furthermore, repeated aspiration can be performed easily, if necessary. We suggest that needle aspiration may be performed as the first-line treatment option for peritonsillar abscess. If the patient does not respond well to needle aspiration, incision and drainage can then be employed.

\section{CONCLUSIONS}

For peritonsillar abscess, needle aspiration as well as incision and drainage provided comparable results in the length of hospital stay. Favourable outcomes were achieved for both groups without any adverse events. Since needle aspiration is easy to perform and inflicts less pain on the patients, we suggest that needle aspiration may be performed as the firstline treatment of peritonsillar abscess. Incision and drainage may be performed later if the patients do not respond well.

\section{REFERENCES}

1. Brodsky L, Poje C. Tonsillitis, tonsillectomy and adenoidectomy. In: Bailey BJ, Johnson JT, Newlands SD, eds. Head and Neck Surgery Otolaryngology. $4^{\text {th }}$ ed. Philadelphia: Lippincott Williams \& Wilkins; 2006: 1183-1198

2. Habib M, Fazal-e-Wahid, Ullah I, Iqbal J, Ullah S. Peritonsillar abscess: is incision drainage superior to abscess tonsillectomy. Ann Pak Inst Med Sci 2011; 7: 62-4.

3. Khan MI, Iqbal K, Muhammad. Peritonsillar abscess: comparison of outcome of incision and drainage versus needle aspiration. Gomal $\mathrm{J}$ Med Sci 2012; 10: 205-8.

4. Edinger JT, Hilal EY, Dastur KJ. Bilateral peritonsillar abscesses: a challenging diagnosis. Ear Nose Throat J 2007; 86: 162-3.

5. Alroud SA, Aljfout QM, Almomani S, Nahleh ELA, Alnawaiseh ST, Alrawashdeh MI. Peritonsillar abscess management; needle aspiration versus incision and drainage, comparison of outcomes. Int J Biol Med Res 2012; 3: 2545-7.

6. Tyagi V, Kaushal A, Grag D, De S, Nagpure P. Treatment of peritonsillar abscess - a prospective study of aspiration verses incision and drainage. Calicut Med J 2011; 9: e3

7. Khan Q, Hamza A, Haleem G, Fazal-e-Wahid. Comparative study of management of peritonsillar abscess by needle aspiration versus incision and drainage. Pak J Med Health Sci 2011; 5: 699-701.

8. Herzon FS. Peritonsillar abscess: incidence, current management practices, and a proposal for treatment guidelines. Laryngoscope 1995; 105: $1-17$.
Submitted 05 Sep 2013

Accepted 20 Jan 2014

Published 19 Mar 2014

Online: http://myspot.mona.uwi.edu/wimjopen/article/51

(c) Chi et al 2014.

This is an open access article made freely available under Creative Commons Attribution 4.0 International (CC BY 4.0). Users are free to share, copy and adapt this work as long as the copyright holder (author) is appropriately and correctly credited. See http://creativecommons. org/licences/by/4.0/deed.en us for more information. 